\title{
THE DREYER AND WARD SIGMA REACTION IN CONGENITAL SYPHILIS
}

BY

\author{
HUGO E. HALL, M.B., B.Сн.
}

Clinical Assistant to the V.D. Department, Royal Victoria Hospital, Belfast; AND

FREDK. M. B. ALLEN, M.D., M.R.C.P.,

Assistant Physician, Belfast Hospital for Sick Children; Medical Registrar, Royal Victoria Hospital, Belfast.

In a report to the Medical Research Council ( ${ }^{1}$ ) of 1,342 cases examined by the Harrison, Fleming and Dreyer methods, Houston, Campbell and Smyth working in the same Unit showed the accuracy and greater sensitiveness of the Sigma technique in the diagnosis of syphilis. These investigators also found that " the flocculation test of Dreyer and Ward was an interesting and valuable method of determining the progress of a case of syphilis under treatment."

Since 1921, 52 cases of congenital syphilis'attending the V.D. Department of the Royal Victoria Hospital and referred from the Belfast Hospital for Sick Children have been submitted to this test. All the cases have not been examined by the Dreyer method owing to the difficulty in many instances of obtaining the necessary quantity of blood $(7 \mathrm{cc}$.$) . Otherwise there has not$ been any selection of the cases.

It was noted that all cases of clinical congenital syphilis showed a definitely positive Sigma reading, i.e., greater than 1.5, and that the Wassermann reaction by the Harrison and Fleming methods was also positive. It was also seen that no case had a Sigma reading of 0 even after prolonged treatment, extending over five years in some cases. A few readings of a value under 2 were, however, recorded. The general tendency was for the Sigma to assume a lower figure in response to treatment.

Of the 52 cases considered, 24 were suffering from eye affections. This proportion $\left(46.1 \%\right.$ ) almost coincides with that noted by Jewesbury $\left({ }^{2}\right)$, viz., 42 out of a total of 84 . It is possible that this high percentage is due to the desire for treatment for affections which cause more physical discomfort than many of the other manifestations of the disease. Of these 24 cases, 20 showed a Sigma reading over 10,17 were over 30 , and 5 were over 200 , when first presented for treatment.

There were four cases of iritis with the Sigma ranging from 59 to 94 . One of these was under treatment for 26 months and by then had a reading of 1.8. In another, as a result of the first month's treatment, the Sigma fell from 94 to 33 . This feature of a marked preliminary fall was noted in many cases in the series, and was usually followed by a much more gradual decline in the succeeding months. The accompanying illustration represents this 
graphically. There was one case of iridocyclitis under observation for almost five years, the Dreyer reaction being done 13 times. In spite of treatment the Sigma did not alter materially, although the clinical condition improved

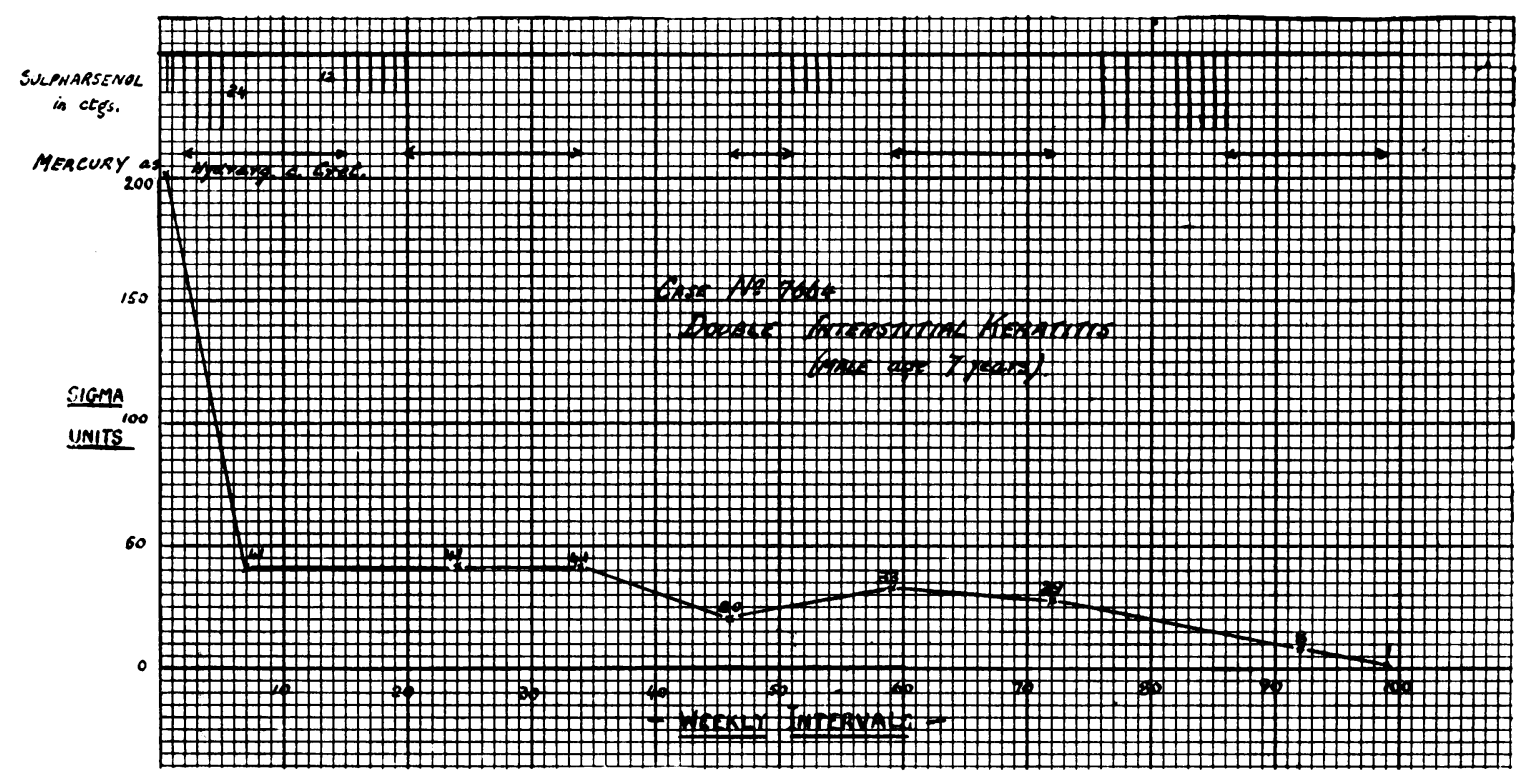

Chart showing the Sigma readings at weekly intervals in a case of Congenital Syphilis undergoing treatment: a rapid fall is followed by a more gradual decline.

decidedly, the eyes being reported "clear" after 23 months' treatment. There was one child with maculx who had a Sigma of 9 eventually reduced to 3. Clinically she was reported "improved."

There were 13 examples of interstitial keratitis, 9 in girls, the ages ranging from 6 to 17 years, 7 of them being in the age group $6-8$ years. The highest Sigma reading was 314 , other high readings being 201 , 190, and 115. One had a reading of 6 when the first Sigma was obtained, the eyes were reported "clear" in 12 months, and after a further 12 months' treatment the Sigma was 1.8, i.e., a virtual negative. The Harrison and Fleming tests were negative. One of these cases is set out in the form of a graph showing how the Sigma was lowered in the course of 105 weeks' treatment, from 201 to J, the Harrison and Fleming being negative at the end of that period. In another case the fall was from 190 to 16 after two months' treatment; in another from 115 to 57 after nine months. To demonstrate how difficult it is to obtain a low Sigma, Case 6,343 is of interest. The first Sigma obtained was 45 in June, 1924, after two months it was 15 , and in spite of treatment varied between 9 and 15 until February 1926, when it was 7. In September 1926, it was still 7, although the clinical condition was reported as " much improved." Another feature noted was that the lower the original Sigma, the less spectacular the immediate 
response. In Case 3,930, in June, 1923, the Sigma was 14.8, the next record was 6.8 and in February, 1926, it was 3.6 and in May, 1926, 2.2 ; the Harrison and Fleming being ++ throughout.

In the series, two cases of condylomata occurred, one in a female of 13 years, and the other in a male infant of two years. In the older patient the Sigma was 79 in June, 1922, and in three months it was 2, with a negative Harrison and positive Fleming. The infant had an original Sigma of 384, and after five months' treatment it was 65 .

In an infant of one month, who had "snuffles" and whose father was known to have syphilis, blood was obtained from the superior sinus and the Sigma was found to be 100 . After a year's treatment it was 6.9 with Harrison+ and Fleming + .

There were three cases in which the retina or choroid was affected, the Sigma readings being 83,70 , and 8.3 . In two of these the figure reached a low level after 16 months' treatment. In the third case where the Sigma was 8.6, after two months it was 16 , and it then gradually subsided to 1.6 in seven months with a negative Harrison and Fleming. This preliminary rise after the initial treatment was noted in a number of cases, and may possibly be explained as a modified Herxheimer reaction.

Ulceration of the mouth, palate and tonsil, and nasal necrosis were diagnosed in 13 cases referred to the department for treatment. The ages varied from 9 to 17 years, 7 being in males. The Sigma reading in this group was rather high ; in six cases it was over 100, and in one 930. As a result of treatment much lower figures were obtained, but only after a lengthy period. Clinically the local lesions cleared up rapidly, but in no case did the Sigma register 0 . In three cases the Sigma was reduced to a figure less than 4 . One case $(2,381)$ was under observation and treatment for over five years. In November, 1921, the Sigma was 118, in six months it was 53 . There was no material change for twelve months, 44 being the figure in March, 1923. In June, 1924, it was 22, and two years and three months later it was 32 , and in February, 1927, 12.2. In all 18 readings were obtained, the Harrison and Fleming being positive throughout. In Case 6,378 where the Sigma was 930, the result of two years' treatment was its reduction to 120 .

There were nine cases of bone and joint affections (" sabre tibia," periostitis, arthritis) at ages varying from 6 to 16 years. The Sigma ranged from 19 to 279 on first observation, but on the whole, the response to treatment was not marked. Clinically there was a decided improvement in the local lesions, especially where there was ulceration and arthritis. In Case 5,349 where the first figure was 279 there was a steady fall to 7 in thirteen months.

Two cases were referred to the department because of stunted growth and anæmia. In one case the spleen was enlarged. These cases also showed a somewhat lower figure eventually, but the striking feature was the rapidity of growth which took place in both. 
The remaining three cases were not under constant observation, and in two cases only one Sigma was obtained. One case was a girl of 11 years with interstitial keratitis, deafness and arthritis with a Sigma of 360 , and positive Harrison and Fleming. She had a sister who showed the same congenital manifestations. The father was asked to report for examination, and although he denied infection his blood was examined and found to be negative. The mother had died some years previously of "rheumatism." There were two cases of Hutchinsonian teeth with Sigma readings of 5 and 65.

Owing to the lack of space the amount and nature of the treatment is not shown in this paper, the purpose of which is to show that the Sigma reading is of value from a diagnostic point of view, and indicative of the intensity of the specific toxin. Serial observations over prolonged periods have shown that it requires persistent treatment to reduce the figure to a "virtual negative" of under 1.5. In no case was a figure of 0 recorded either before or as a result of treatment. Attention has already been drawn to the preliminary fall which occurred when treatment was first given, and the more gradual decline later. The higher the original figure the more striking was this preliminary fall. A rise in the Sigma was noted in a number of cases during the progress of treatment, and it is suggested that this may be an indication of a modified Herxheimer reaction. Case 7,644 is set out in the form of a graph indicating the details of the treatment given.

All the Sigma tests in this paper were carried out in the Pathology Unit of the Royal Victoria Hospital, Belfast, and we are indebted.to its Director, Sir Thomas Houston, for permission to uce the figures. Dr. J. C. Rankin, Physician-in-charge of the V.D. Department, has kindly given us access to the clinical records.

\section{REFERENCES.}

1. Med. Res. Council Report, No. 78 (H.M. Stat. Off.).

2. Jewesbury, R., Frit. Jour. Ven. Dis., 1926, II, 323. 